\title{
On the effect of IMF turning on ion dynamics at Mercury
}

\author{
D. C. Delcourt ${ }^{1}$, T. E. Moore ${ }^{2}$, and M.-C. H. Fok ${ }^{2}$ \\ ${ }^{1}$ LPP, UMR7648, CNRS - Ecole Polytechnique, Saint-Maur des Fossés, France \\ ${ }^{2}$ NASA, Goddard Space Flight Center, Greenbelt, Maryland, USA
}

Received: 23 February 2011 - Revised: 9 May 2011 - Accepted: 22 May 2011 - Published: 10 June 2011

\begin{abstract}
We investigate the effect of a rotation of the Interplanetary Magnetic Field (IMF) on the transport of magnetospheric ion populations at Mercury. We focus on ions of planetary origin and investigate their large-scale circulation using three-dimensional single-particle simulations. We show that a nonzero $B_{X}$ component of the IMF leads to a pronounced asymmetry in the overall circulation pattern. In particular, we demonstrate that the centrifugal acceleration due to curvature of the $\boldsymbol{E} \times \boldsymbol{B}$ drift paths is more pronounced in one hemisphere than the other, leading to filling of the magnetospheric lobes and plasma sheet with more or less energetic material depending upon the hemisphere of origin. Using a time-varying electric and magnetic field model, we investigate the response of ions to rapid (a few tens of seconds) re-orientation of the IMF. We show that, for ions with gyroperiods comparable to the field variation time scale, the inductive electric field should lead to significant nonadiabatic energization, up to several hundreds of eVs or a few keVs. It thus appears that IMF turning at Mercury should lead to localized loading of the magnetosphere with energetic material of planetary origin (e.g., $\mathrm{Na}^{+}$).
\end{abstract}

Keywords. Magnetospheric physics (Planetary magnetospheres)

\section{Introduction}

With an orbit comprised between $\sim 0.3$ and $\sim 0.47$ astronomical units, Mercury is located in a region of the Parker's spiral where the radial component of the Interplanetary Magnetic Field (IMF) is dominant. Because of its appreciable though weak (about 1000 times smaller than that of Earth) intrinsic magnetic field, a miniature magnetosphere forms around

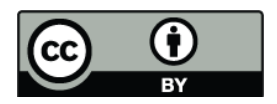

Correspondence to: D. C. Delcourt (dominique.delcourt@lpp.polytechnique.fr) the planet (e.g., Ness, 1979; Russell and Walker, 1985; Anderson et al., 2008) that is expected to be very dynamical and subjected to prominent reconfigurations due to variations of the IMF or variations of the solar wind dynamical pressure (e.g., Slavin et al., 2010). In a recent study (Delcourt et al., 2010), we examined the response of magnetospheric ions to compression of the magnetosphere due to abrupt solar wind pressure increase. In that study, we focused on ions originating from the planetary exosphere that are suspected to significantly contribute to the inner hermean magnetotail as evidenced from MESSENGER data (Zurbuchen et al., 2008). We showed that, during such events, a mass selective nonadiabatic heating is at work due to the inductive electric field. In the present study, we investigate the effect of IMF re-orientation on the dynamics of magnetospheric ions and show that a similar heating should operate. In Sect. 2, we describe the procedure adopted to simulate the ion transport during turning of the IMF. In Sect. 3, we discuss some results of trajectory computations in steady state, while those obtained during dynamical reconfigurations of the magnetosphere are presented in Sect. 4.

\section{Modeling of magnetosphere reconfiguration}

To examine the response of magnetospheric ions to IMF turning at Mercury, we performed single-particle simulations in three-dimensional time-varying electric and magnetic fields that provide a first-order description of the hermean environment. As for the magnetic field, it was accounted for using a modified version of the model of Luhmann and Friesen (1979) (hereinafter referred to as LF-79). This simple model that reproduces basic features of the magnetosphere, consists of a planetary dipole field and an infinite Harris-type current sheet (see Delcourt et al., 2010). In the present study, we take for the planetary dipole moment $M=250 \mathrm{nT} R_{M}^{3}$ (e.g., Anderson et al., 2008). We also

Published by Copernicus Publications on behalf of the European Geosciences Union. 


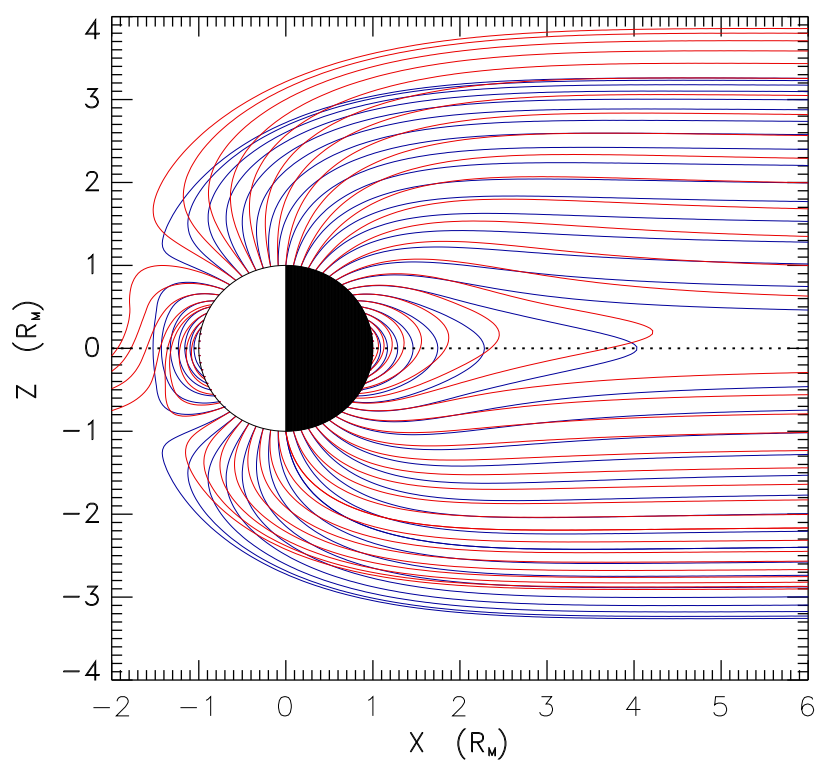

Fig. 1. Magnetic field lines in the noon-midnight meridian plane for IMF $B_{\mathrm{X}}=0$ and IMF $B_{\mathrm{X}}=20 \mathrm{nT}$ (color-coded in blue and red, respectively). The latitude of the magnetic field line foot on the planet varies by step of $5^{\circ}$. The $\mathrm{X}$-axis points toward the tail, the Y-axis, from dusk to dawn.

assume a current sheet field, $B_{T}$, and half-thickness, $L$, of $50 \mathrm{nT}$ and $0.5 R_{M}$, respectively, which leads to a subsolar magnetopause near $1.5 R_{M}$. On the other hand, we simulate the effect of the IMF by superposing a $B_{\mathrm{X}}$ component to the above modified LF-79. Specifically, we consider a situation where the IMF $B_{\mathrm{X}}$ varies from 0 up to $20 \mathrm{nT}$. The magnetospheric configurations obtained in the initial and final states are shown in Fig. 1 that presents magnetic field lines in the noon-midnight meridian plane (in this figure as well as in the following, note that the $\mathrm{X}$-axis points in the antisunward direction, and the Y-axis, from dusk to dawn). The warping of the magnetosphere that results from a non-zero IMF $B_{\mathrm{X}}$ is clearly apparent from Fig. 1.

Like in previous studies (e.g., Delcourt et al., 2002, 2010), the large-scale magnetospheric convection at Mercury is modeled by assuming a situation similar to that at Earth, with a two-cell pattern of potential distribution in the immediate vicinity of the planet as featured in the Volland-Stern description (see, e.g., Volland, 1978). The inclusion of a nonzero IMF $B_{\mathrm{X}}$ however leads us to implement some modification. Indeed, in the initial state with IMF $B_{X}=0$ (blue lines in Fig. 1), the symmetry between Northern and Southern Hemispheres allows us to use the same potential distribution in both hemispheres. This is not the case in the final state with IMF $B_{X}=20 \mathrm{nT}$ (red lines in Fig. 1). In this latter case, distinct potential distributions must be considered which would otherwise lead to unrealistically abrupt variations of the electric field (hence, unrealistic variations of the particle magnetic moment) at the transition between one hemisphere and
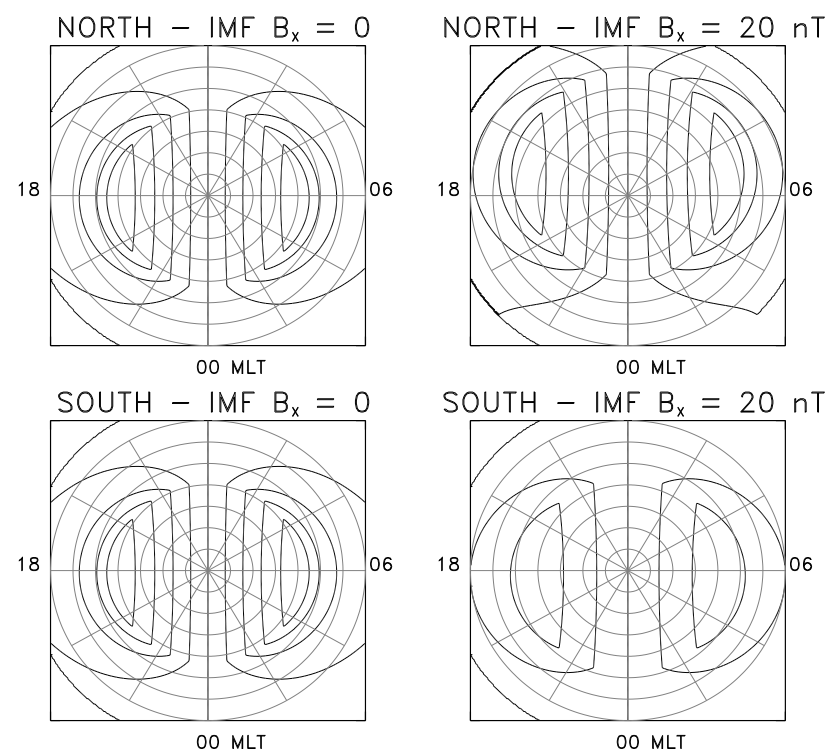

Fig. 2. Potential distribution in (top) Northern and (bottom) Southern Hemispheres for (left) IMF $B_{X}=0$ and (right) IMF $B_{X}=20 \mathrm{nT}$. Circles indicate latitudes by steps of $10^{\circ}$. Equipotentials are shown by steps of $2 \mathrm{kV}$.

the other. The potential distribution in the Volland (1978) model is characterized by two parameters, viz., (i) the colatitude of the Convection Reversal Boundary (CRB) above and below which plasma is flowing in antisunward and sunward directions, respectively, (ii) the cross-polar cap potential drop. As for the CRB colatitude, it is evaluated at each step of the simulation by calculating in each hemisphere the latitude of the last closed field line in the noon meridian plane. For the case shown in Fig. 1 (red lines), this colatitude is of the order of $58^{\circ}$ and $43^{\circ}$ in Northern and Southern Hemispheres, respectively. As for the cross-polar cap potential drop, for a given value in the Northern Hemisphere, the value in the opposite hemisphere is calculated in such a manner that the $\boldsymbol{E} \times \boldsymbol{B}$ drift speeds obtained in the distant magnetotail from one and the other potential distributions are identical. The resulting distributions can be appreciated in Fig. 2 that shows equipotentials in both hemispheres (top and bottom rows) in the initial and final states (left and right panels). While the left panels (IMF $B_{\mathrm{X}}=0$ ) of Fig. 2 display similar patterns, notable differences can be seen in the right panels for IMF $B_{\mathrm{X}}=20 \mathrm{nT}$, with tailward flow over a wider latitudinal range in the Northern Hemisphere (top) than in the southern one (bottom).

Figure 2 relates to the convection electric field that results from coupling with the solar wind. On the other hand, the transition from IMF $B_{\mathrm{X}}=0$ to IMF $B_{\mathrm{X}}=20 \mathrm{nT}$ gives rise to an inductive electric field that must also be accounted for. This very transition was modeled in a like manner to Delcourt et al. (2010). At a given position $\boldsymbol{r}$ and a given time $t$ of the transition, the magnetic field was obtained as: 

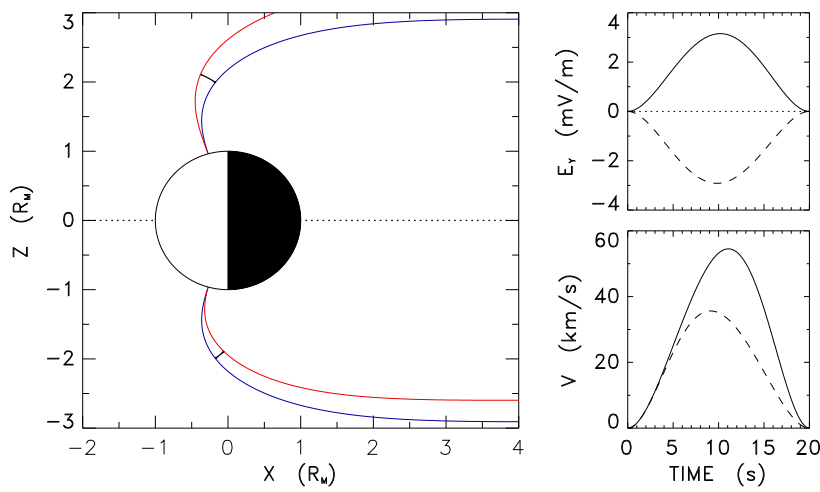

Fig. 3. (Left) Evolution of a given magnetic field line from IMF $B_{\mathrm{X}}=0$ (blue color code) to IMF $B_{\mathrm{X}}=20 \mathrm{nT}$ (red color code). In both hemispheres, the black line shows the $\boldsymbol{E} \times \boldsymbol{B}$ drift path of a zero energy particle during the IMF turning. (Right top) Electric field and (right bottom) $\boldsymbol{E} \times \boldsymbol{B}$ drift speed versus time. The time scale of the IMF turning is set to $20 \mathrm{~s}$.

$\boldsymbol{B}(\boldsymbol{r}, t)=\boldsymbol{B}_{o}(\boldsymbol{r})+f(t)\left[\boldsymbol{B}_{f}(\boldsymbol{r})-\boldsymbol{B}_{o}(\boldsymbol{r})\right]$

where $\boldsymbol{B}_{o}$ and $\boldsymbol{B}_{f}$ correspond to initial and final configurations, respectively. In Eq. (1), $f(t)$ describes the rate of change of the magnetic field. As described in Delcourt et al. (2010), imposing a zero induced electric field as well as its zero slope at the onset and at the end of the magnetic transition leads to a polynomial of degree 5 for $f(t)$. Denoting by $\tau_{B}$ the time scale of the magnetic transition, this polynomial smoothly varies between 0 at $t=0$ and 1 at $t=\tau_{B}$.

As for the time-dependent electric field, it was considered to be the sum of two contributions. The first contribution is that induced by the time varying magnetic field, viz., $\boldsymbol{E}=-\partial \boldsymbol{A} / \partial t$ where the vector potential $\boldsymbol{A}$ is such that $\mathrm{curl}$ $(\boldsymbol{A})=\boldsymbol{B}$ as given by Eq. (1). Since the vector potential is undetermined to the gradient of a scalar field, a second contribution $\nabla \Phi$ is also considered that cancels the parallel component of the induced electric field, that is, $(\partial \boldsymbol{A} / \partial t) \cdot \boldsymbol{B}=0$. The calculation of this second contribution is described in Appendix B of Delcourt et al. (1990). As discussed by Heikkila and Pellinen (1977), this latter contribution actually redistributes the electric field in the perpendicular direction and accounts for plasma polarization. At a given position $\boldsymbol{r}$ and time $t$, the electric field due to IMF turning is thus obtained as:

$\boldsymbol{E}(\boldsymbol{r}, t)=-[\partial \boldsymbol{A}(\boldsymbol{r}, t) / \partial t]-\nabla \Phi_{\mathrm{pol}}(\boldsymbol{r}, t)$

where $\nabla \Phi_{\text {pol }}$ is the electric potential due to the free charges, and $\boldsymbol{A}$ is the instantaneous vector potential such that:

$\boldsymbol{A}(\boldsymbol{r}, t)=\boldsymbol{A}_{o}(\boldsymbol{r})+f(t)\left[\boldsymbol{A}_{f}(\boldsymbol{r})-\boldsymbol{A}_{o}(\boldsymbol{r})\right]$

where $\boldsymbol{A}_{o}$ and $\boldsymbol{A}_{f}$ relate to initial and final configurations, respectively.

The electric and magnetic field variations obtained from Eqs. (1-3) can be appreciated in Fig. 3 that shows the evolution of two field lines anchored at the same latitude $\left(75^{\circ}\right)$ in the Northern and Southern Hemispheres. In the left panel of Fig. 3, it can be seen that, as a result of the IMF $B_{X}$ growth, the field line in the Northern Hemisphere is rapidly brought sunward while that in the Southern Hemisphere is pulled tailward. Note that, in this figure as well as in the following simulations, the time scale $\tau_{B}$ of the magnetic transition was set to $20 \mathrm{~s}$. Because propagation effects are not accounted for in the present model, this $20 \mathrm{~s}$ time scale describes the duration of the local magnetic field reconfiguration, the overall reconfiguration of the magnetosphere itself occurring on a larger time scale (a few minutes). To further illustrate the electric field variation during this magnetic transition, the black lines in the left panel of Fig. 3 show the trajectories of zero energy particles that experience only $\boldsymbol{E} \times \boldsymbol{B}$ drift from initial to final configurations. The right panels of Fig. 3 show the electric field and $\boldsymbol{E} \times \boldsymbol{B}$ drift speed along these paths. Consistently with the field line evolution displayed in the left panel, a dawnward induced electric field is obtained in the Northern Hemisphere (solid line) with a peak magnitude of $\sim 3 \mathrm{mV} \mathrm{m}^{-1}$; hence, a peak $\boldsymbol{E} \times \boldsymbol{B}$ drift speed of $\sim 50 \mathrm{~km} \mathrm{~s}^{-1}$ (average value of the order of $30 \mathrm{~km} \mathrm{~s}^{-1}$ ). In the Southern Hemisphere, a duskward induced electric field is obtained that leads to a peak $\boldsymbol{E} \times \boldsymbol{B}$ drift speed somewhat smaller than in the Northern Hemisphere, of the order of $35 \mathrm{~km} \mathrm{~s}^{-1}$.

Once electric and magnetic fields were defined, test ions were traced throughout Mercury's magnetosphere using the full equation of motion (integrated by means of a fourthorder Runge-Kutta). Such a tracing technique is fairly standard and has been used for instance in a variety of studies of plasma transport in the terrestrial magnetosphere. In order to examine the impact of changes in the solar wind on the large-scale magnetospheric circulation, some of these studies do not use analytical or semi-empirical fields but results of MHD simulations (e.g., Moore et al., 2005; Peroomian and El-Alaoui, 2008). Such an approach is also used for specific case studies (e.g., Ashour-Abdalla et al., 2010). In this latter approach, the electric and magnetic fields at the test particle position are interpolated from the three-dimensional grid of the MHD simulations. The aim of the present study is not to examine specific solar wind cases but to investigate from a general perspective some transport features associated with IMF rotation; hence, the use of a simple analytical description for both electric and magnetic fields. It should be noted that, be they analytical or MHD-based, both approaches are not self-consistent in the sense that there is no iteration and feedback between the calculated particle motion and the field. Such approaches are nonetheless useful to understand the complexity of magnetospheric particle dynamics. In this regard, the three-dimensional electric and magnetic fields used in the present study reproduce basic magnetospheric features expected at Mercury, with antisunward transport from the cusp region over the polar cap and return flow toward the sun at low latitudes. Note also that the present trajectory computations do not include smallscale acceleration processes. The energization affecting ions 

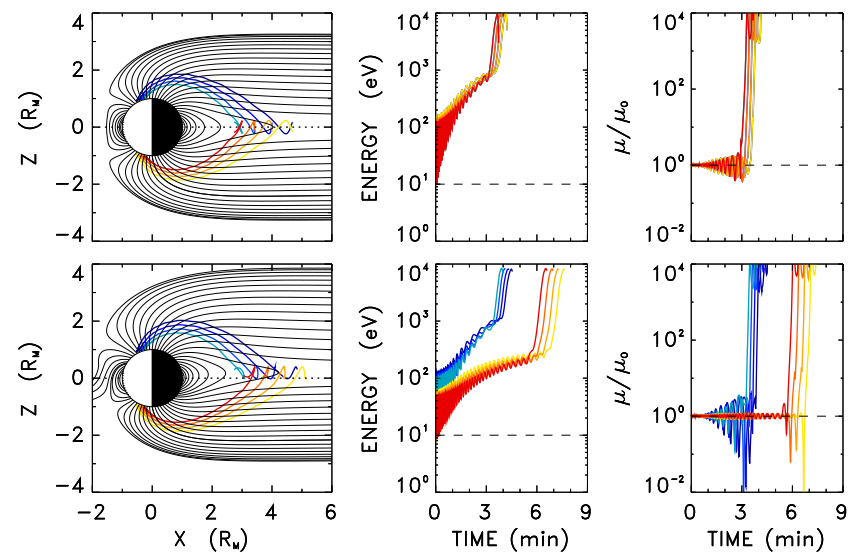

Fig. 4. Model $\mathrm{Na}^{+}$trajectories for (top) IMF $B_{X}=0$ and (bottom) IMF $B_{X}=20 \mathrm{nT}$. (from left to right) Trajectory projections in the $\mathrm{X}-\mathrm{Z}$ planes, energy, and magnetic moment (normalized to the initial value) as a function of time. The ions are launched with $10 \mathrm{eV}$ energy and $30^{\circ}$ pitch angle on the noon meridian and from different latitudes (from $60^{\circ}$ to $75^{\circ}$ by steps of $5^{\circ}$ ) in the Northern Hemisphere (blue color code) and in the southern one (yellow-red color code).

during their three-dimensional transport is solely due to the large-scale convection electric field as well as to the surging electric field associated with IMF rotation (the role of gravity being negligible at Mercury). The modeling technique adopted here (Eqs. 1-3) ensures that the parallel electric field vanishes regardless of the particle position in the magnetosphere. This parallel electric field is not arbitrarily set to zero. As described above, the condition $\boldsymbol{E} \cdot \boldsymbol{B}=0$ is achieved by assuming that, in first approximation, magnetic field lines are equipotential and by making use of a polarization electric field, $\nabla \Phi_{\mathrm{pol}}$, that redistributes the field in the perpendicular direction (see Appendix B of Delcourt et al. (1990) for a detailed description of the modeling technique). As will be shown in Sect. 4 hereinafter, explicit calculation of the parallel component of the three-dimensional electric field demonstrates that it is indeed negligible (less than $10^{-4} \mathrm{mV} \mathrm{m}^{-1}$ along the computed ion trajectories).

\section{Centrifugal acceleration in magnetospheric lobes}

In the adiabatic (guiding center) approximation, the parallel equation of motion in the absence of parallel electric field and neglecting the gravitational acceleration writes (see, e.g., equation 1.20 of Northrop, 1963):

$\dot{V}_{/ /}=\boldsymbol{V}_{E} \cdot\left[\frac{\partial \boldsymbol{b}}{\partial t}+V_{/ /} \frac{\partial \boldsymbol{b}}{\partial s}+\boldsymbol{V}_{E} \cdot \nabla \boldsymbol{b}\right]-\frac{\mu}{m} \frac{\partial B}{\partial s}$

Here, $\boldsymbol{V}_{E}$ is the $\boldsymbol{E} \times \boldsymbol{B}$ drift velocity, $V_{/ /}$, the parallel speed of the guiding center, $\boldsymbol{b}$, a unit vector in the $\boldsymbol{B}$ direction, $s$, the curvilinear abscissa along the field line, and $m$ and $\mu$,
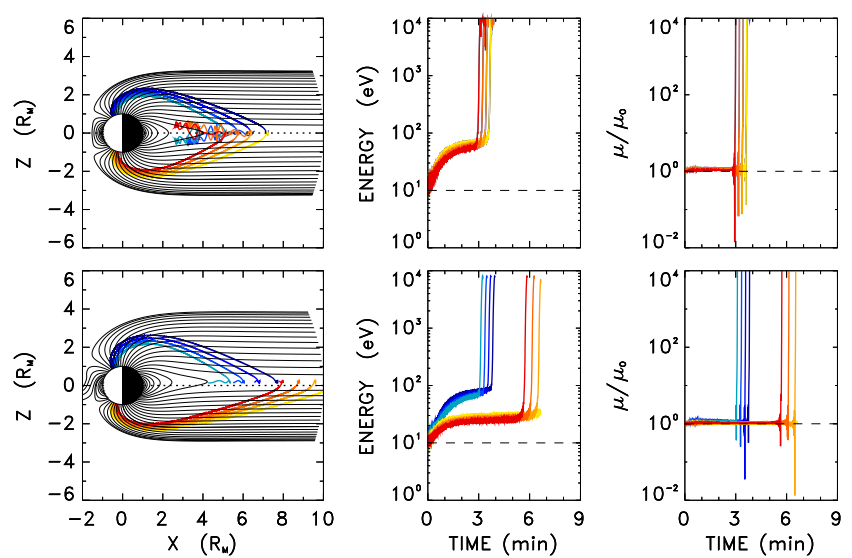

Fig. 5. Identical to Fig. 4 but for protons.

the particle mass and magnetic moment, respectively. Equation (4) displays two distinct curvature related acceleration terms, viz., the second term on the right hand side of Eq. (4) is due to curvature of the magnetic field lines which can lead to prominent acceleration upon crossing of a field reversal as is the case in the magnetotail current sheet. The third term on the right hand side of Eq. (4) is a centrifugal acceleration that depends upon curvature of the $\boldsymbol{E} \times \boldsymbol{B}$ drift paths. In the Earth's magnetosphere, this centrifugal effect can lead to upward ion energization of a few eVs or a few tens of eVs at low altitudes (e.g., Cladis, 1986) or to focusing (referred to as "centrifugal trapping") of slow plasma sheet particles into the equatorial region (e.g., Delcourt et al., 1993).

It was shown in Delcourt et al. (2002) that, because curvature radii of the $\boldsymbol{E} \times \boldsymbol{B}$ drift paths are much smaller at Mercury than at Earth, the above centrifugal effect (third term in Eq. 4) is enhanced and may lead to quite substantial parallel energization during transport from high to low latitudes. As a matter of fact, it was demonstrated that, as a result of this effect, heavy ions originating from Mercury's exosphere (e.g., $\mathrm{Na}^{+}$) may experience energization of several tens or a few hundreds of $\mathrm{eV}$ within minutes, suggesting a loading of the magnetospheric lobes more rapid and more energetic than at Earth. This efficient centrifugal energization of lowenergy planetary material can be appreciated in Figs. 4 and 5 that present results of trajectory computations for both $\mathrm{Na}^{+}$ and $\mathrm{H}^{+}$, respectively. In these figures, test ions are launched from different latitudes (from $60^{\circ}$ to $75^{\circ}$ by steps of $5^{\circ}$ ) in both hemispheres in the dayside sector.

The top rows of Figs. 4 and 5 show the results obtained for IMF $B_{\mathrm{X}}=0$. In this case, symmetrical patterns are obtained regardless of the hemisphere of origin. Upon transport into the lobes, $\mathrm{Na}^{+}$ions experience a net energy gain of several hundreds of $\mathrm{eV}$ (top center panel of Fig. 4) while that of protons is of the order of $60 \mathrm{eV}$ (top center panel of Fig. 5). Subsequently, the ions intercept the equatorial neutral sheet where their motion turns nonadiabatic as evidenced from the 

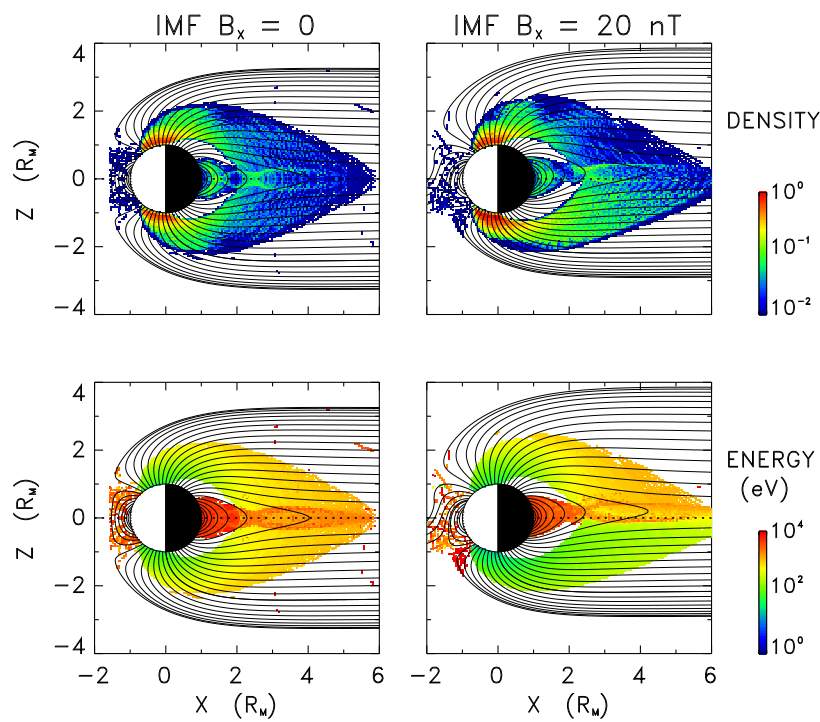

Fig. 6. (top) Model normalized density and (bottom) average energy of $\mathrm{Na}^{+}$ions in the noon-midnight meridian plane for (left) IMF $B_{\mathrm{X}}=0$ and (right) IMF $B_{\mathrm{X}}=20 \mathrm{nT}$. Density and energy are coded according to the color scales at right.

large $\mu$ changes in the rightmost panels. During this nonadiabatic sequence that is due to the small spatial scale of the magnetic field as compared to the ion Larmor radius (e.g., Speiser, 1965; Chen and Palmadesso, 1986; Büchner and Zelenyi, 1989), $\mathrm{Na}^{+}$rapidly travel westward and are eventually lost into the dusk magnetopause. As for protons, they remain temporarily trapped at low latitudes before intercepting the dusk magnetopause. For both $\mathrm{Na}^{+}$and protons, it can be seen that this rapid westward drift toward the dusk magnetopause goes together with prominent energization, as apparent from the abrupt energy increase near the end of the trajectories in the center panels of Figs. 4 and 5. During oscillation about the equator and simultaneous duskward transport, the net energy gain realized via crossing of the convection electric field equipotentials is of the order of $10 \mathrm{kV}$, i.e., half of the cross-tail potential drop.

Because it introduces a hemispherical asymmetry, we expect significant deviations from these results in the case of a nonzero IMF $B_{\mathrm{X}}$. This is illustrated in the bottom rows of Figs. 4 and 5 for $\mathrm{Na}^{+}$and $\mathrm{H}^{+}$, respectively. In this latter case, it can be seen that, regardless of mass, the test ions originating from the southern high-latitude dayside sector (trajectories coded in yellow-red) travel somewhat further out into the magnetotail. Most notably, the $\boldsymbol{E} \times \boldsymbol{B}$ related centrifugal energization realized along these latter paths is significantly lesser than that of ions originating from the Northern Hemisphere (trajectories coded in blue), of the order of a few hundreds of $\mathrm{eV}$ instead of $\sim 1 \mathrm{keV}$ for $\mathrm{Na}^{+}$(Fig. 4), and a few tens of eVs instead of $\sim 100 \mathrm{eV}$ for protons (Fig. 5). The net time of flight from the planet surface until the nightside
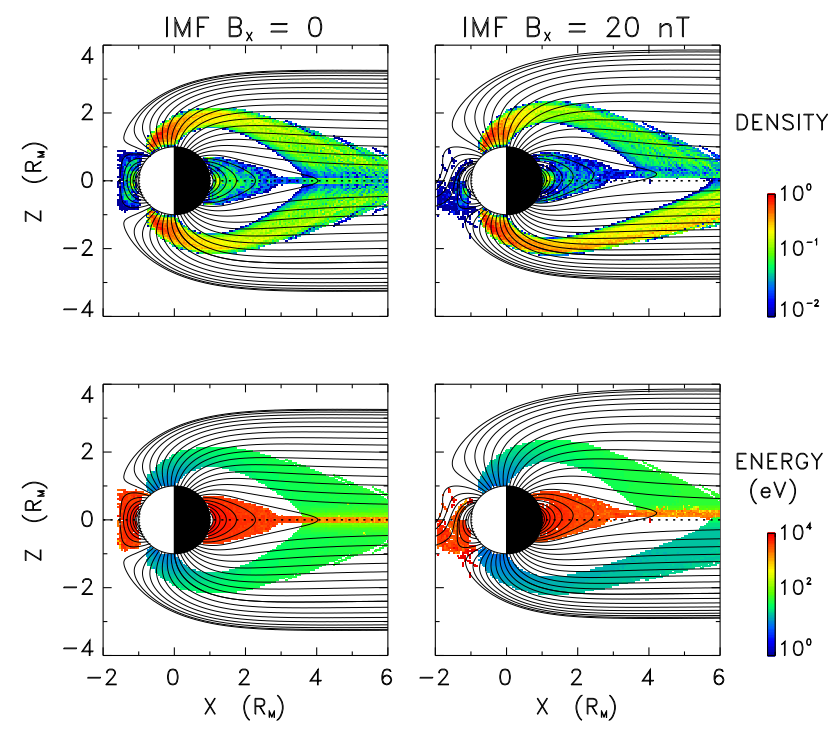

Fig. 7. Identical to Fig. 6 but for protons.

neutral sheet is accordingly larger for Southern Hemisphere originating ions.

A more general view of these different centrifugal effects can be obtained from Figs. 6 and 7 that show the results of systematic trajectory computations of $\mathrm{Na}^{+}$and protons originating from the high-latitude dayside exosphere. The top and bottom panels in these figures show the normalized density and average energy, respectively, for (left) IMF $B_{X}=0$ and (right) IMF $B_{X}=20 \mathrm{nT}$. These figures clearly illustrate the $\boldsymbol{E} \times \boldsymbol{B}$ ion transport all the way from the dayside sector into the equatorial plasma sheet in the nightside sector over the polar cap. Such a large-scale transport leads to a substantial filling of the magnetospheric lobes prior to particle interaction with the tail current sheet. Note the gradual decrease of the lobe density along these paths, by about 2 orders of magnitude as compared to that at low altitudes (top panels). Not surprisingly, symmetrical patterns are obtained in the case of IMF $B_{\mathrm{X}}=0$, with lobe energization up to $\sim 1 \mathrm{keV}$ for $\mathrm{Na}^{+}$and $\sim 100 \mathrm{eV}$ for protons. As expected from Figs. 4 and 5, a nonzero IMF $B_{X}$ leads to a quite distinct pattern with prominent differences between northern and southern lobes. In this latter case, regardless of ion mass (Figs. 6 and 7), the northern lobe exhibits significantly lesser density and larger average energy (equivalently, smaller loading time) than the southern one, by about one order of magnitude. Also, because protons originating from the Southern Hemisphere intercept the equator further out in the magnetotail, a lesser $\mathrm{H}^{+}$filling of the innermost magnetosphere is obtained. Finally, regardless of the IMF orientation, note the prominent energization (up to several keVs) realized upon nonadiabatic interaction of the ions with the tail neutral sheet; hence, the build-up of a layer of energetic material. 

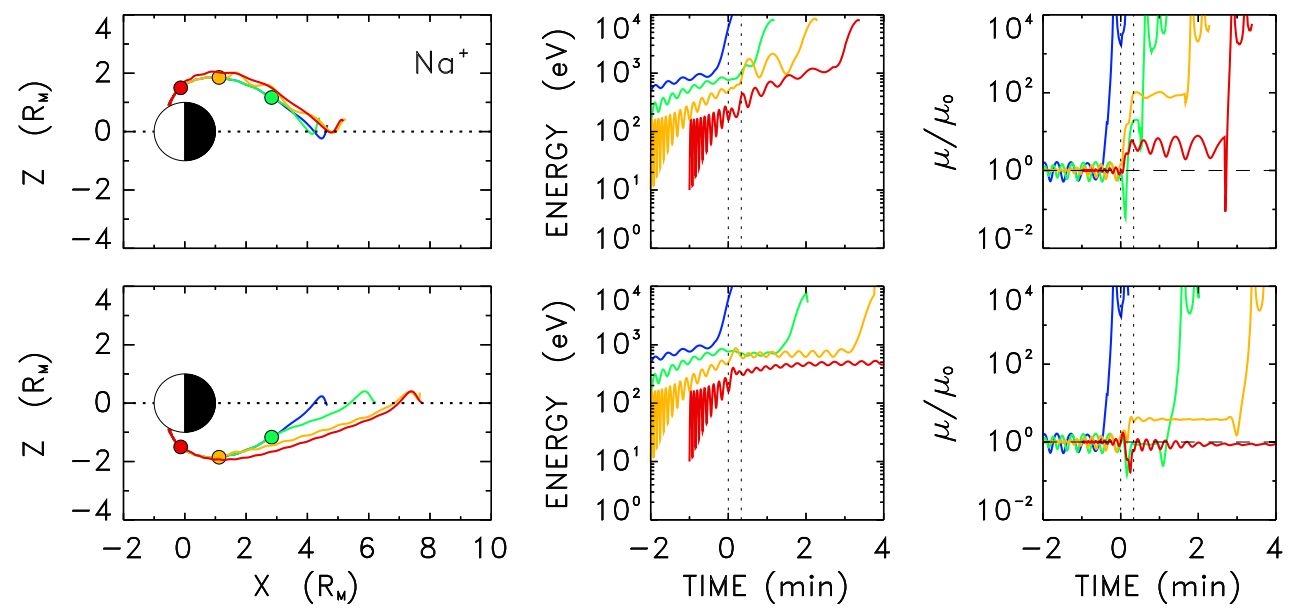

Fig. 8. Model $\mathrm{Na}^{+}$trajectories during a 20-s turning of the IMF from $B_{X}=0$ to $B_{X}=20 \mathrm{nT}$. The test ions are launched from the same location $\left(60^{\circ}\right.$ latitude on the noon meridian) with $10 \mathrm{eV}$ energy and $30^{\circ}$ pitch angle. Top and bottom panels relate to ions originating from Northern and Southern Hemispheres, respectively. (From left to right) Trajectory projections in the X-Z plane, energy, and magnetic moment (normalized to the initial value) as a function of time (measured from the onset of the IMF turning). The different colors correspond to distinct times of ejection (by steps of $1 \mathrm{~min}$ ). Color-coded closed circles in the left panels show the position of the particles at the onset of the IMF turning. Vertical dotted lines in the right panels delimit the 20-s magnetic transition.

\section{Ion dynamics during IMF turning}

The results presented in Sect. 3 relate to steady IMF orientations. To illustrate the behavior of ions during turning of the IMF, Figs. 8 and 10 show test ion trajectories using the modeling technique described in Sect. 2, with a gradual transition (over a time scale of $20 \mathrm{~s}$ ) from IMF $B_{\mathrm{X}}=0$ to IMF $B_{\mathrm{X}}=20 \mathrm{nT}$. In these figures, $\mathrm{Na}^{+}$and protons were launched from the same location in the high-latitude dayside sector at distinct (color-coded) times (by steps of $1 \mathrm{~min}$ ) before the onset of the magnetic transition. In the left panel of Figs. 8 and 10, this onset is indicated by (color-coded) closed circles. The top row in these figures show ions launched from the Northern Hemisphere, while the bottom row relates to the southern one.

Looking first at $\mathrm{Na}^{+}$in Fig. 8, it can be seen in the left panels that, after convection over the polar cap into the nightside sector, the test ions reach the equatorial magnetotail where they experience nonadiabatic interaction with the neutral sheet. In this figure, the trajectories coded in blue correspond to ions reaching the equator before the onset of the IMF turning. In this case, similar results are obtained regardless of the hemisphere of origin, be it for the trajectory apex or for the net $\mu$ change. For ions leaving the exosphere at somewhat later times, substantial differences are noticeable. As discussed in Figs. 4 and 5, it can be seen that as a result of the growing IMF $B_{\mathrm{X}}$, the trajectory apex of Southern Hemisphere originating ions gradually moves tailward. For these ions, lesser centrifugal accelerations also are achieved in the magnetospheric lobes. Most notably, in the right panels of Fig. 8, an increase of magnetic moment and concurrent increase of energy can be seen during the magnetic transition.

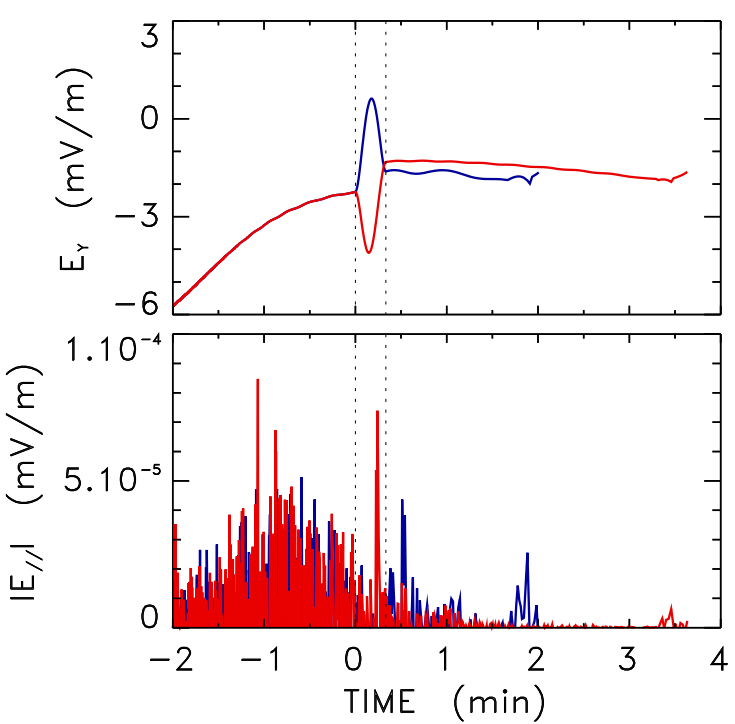

Fig. 9. Electric field variations along the trajectories of $\mathrm{Na}^{+}$ launched $120 \mathrm{~s}$ before the onset of IMF turning (trajectories coded in yellow in Fig. 8): (top) electric field component in the Ydirection, (bottom) parallel electric field (in absolute value) versus time. Blue and red colors correspond to $\mathrm{Na}^{+}$launched form Northern and Southern Hemispheres, respectively. Vertical dotted lines delimit the 20-s magnetic transition.

The $\mu$ increase is most pronounced (by two orders of magnitude) for the $\mathrm{Na}^{+}$located at mid-altitudes in the northern lobe at the onset of the magnetic transition (trajectories coded in green and yellow). This behavior contrasts with that obtained for ions located at low altitude (trajectory coded in red) or in the equatorial region. 

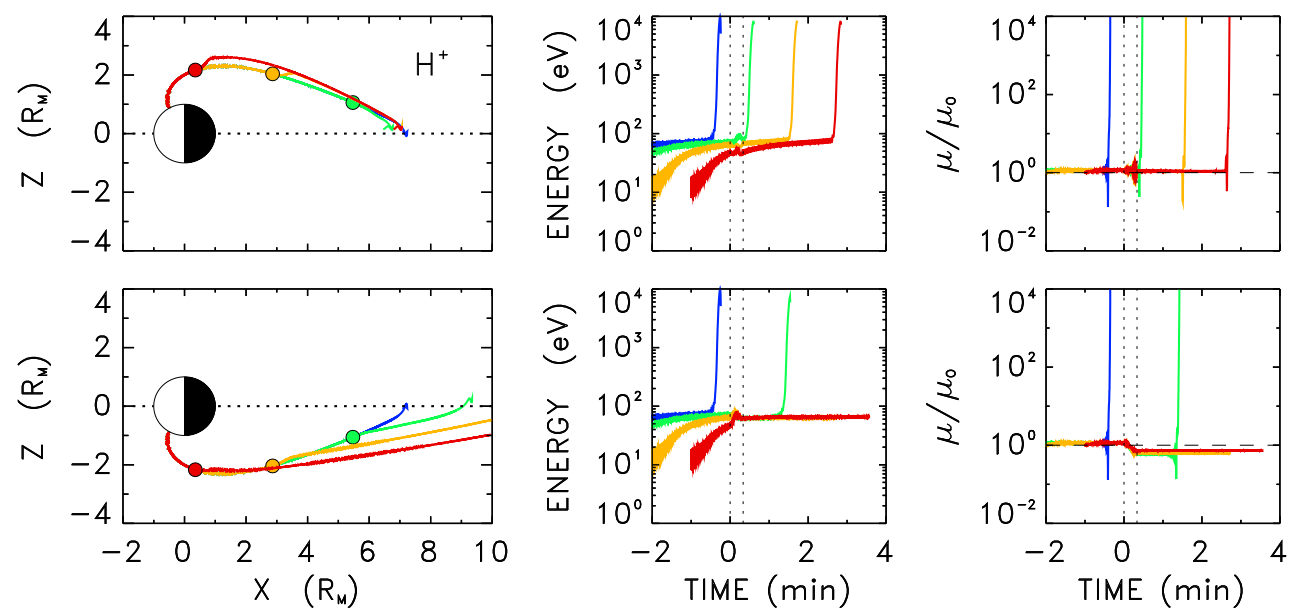

Fig. 10. Identical to Fig. 8 but for protons.

The electric field variations experienced by the ions can be appreciated in Fig. 9 that displays the component in the dawn-dusk direction throughout transport (top panel). The bottom panel of Fig. 9 also shows the magnitude of the parallel component obtained in these computations. The electric field profiles presented here are those obtained for the test $\mathrm{Na}^{+}$launched two minutes before onset of the IMF turning (i.e., trajectories coded in yellow in Fig. 8). Looking at the top panel of Fig. 9, it can be seen that, since one initially has IMF $B_{\mathrm{X}}=0$, identical $E_{\mathrm{Y}}$ variations are first obtained for Northern (coded in blue) and Southern Hemisphere (coded in red) originating ions, with a magnitude up to $\sim 6 \mathrm{mV} \mathrm{m}^{-1}$ at low altitudes. From $t=0$ until $t=20 \mathrm{~s}$, the IMF $B_{\mathrm{X}}$ increases up to $20 \mathrm{nT}$ and rapid $E_{\mathrm{Y}}$ variations of a few $\mathrm{mV} \mathrm{m}^{-1}$ can be seen. These variations lead to a net decrease of the convection electric field in the Northern Hemisphere and a net increase in the southern one, consistently with the magnetic field line motion portrayed in Figs. 1 and 3. Subsequently, the $E_{\mathrm{Y}}$ profiles obtained for the two test $\mathrm{Na}^{+}$are different due to their distinct trajectories (see Fig. 8). On the other hand, the bottom panel of Fig. 9 confirms that the modeling approach adopted in the present study leads to a negligible parallel electric field component throughout transport, with a magnitude in the $10^{-5}-10^{-4} \mathrm{mV} \mathrm{m}^{-1}$ range (equivalently, leading to a net change of parallel energy of at most a few Volts during transport).

A result similar to that displayed in Fig. 8 was reported in the study of Delcourt et al. (2010) that was dedicated to the dynamics of ions during compression of Mercury's magnetosphere (see, e.g., Fig. 3 of that study). It was shown in this latter study that the above nonadiabatic behavior results from resonance between the ion gyromotion and the time-varying magnetic field. That is, for ions with $\tau_{C} \ll \tau_{B}$ ( $\tau_{C}$ being the ion cyclotron period), the magnetic field does not change significantly during a cyclotron turn and the motion is adiabatic ( $\mu$ conserving) (e.g., Northrop, 1963). In Fig. 8, this is the case at low altitudes where the magnetic field is large. On the other hand, for ions with $\tau_{C} \gg \tau_{B}$, the work done by the induced electric field during a fraction of cyclotron turn is not significant enough to yield substantial perpendicular energization, a situation that is achieved for ions located in the equatorial region at onset. In between (i.e., at mid-latitudes), for ions with $\tau_{C} \approx \tau_{B}$, nonadiabatic behavior with possibly large $\mu$ enhancement is obtained due to phasing between the gyromotion and the surging electric field. This nonadiabatic energization is controlled by the magnitude of the peak induced electric field. It thus depends upon $\tau_{B}$ as well as $\boldsymbol{\Delta A}$ (Eq. 3), a shorter time scale or a wider magnetic transition resulting into a larger energy gain.

While the study of Delcourt et al. (2010) investigated the effect of a sudden compression of the magnetosphere (modeled via an abrupt increase of the magnetospheric lobe field, $B_{T}$ ), the present study is focusing on the impact of IMF turning and consequent warping of the magnetosphere. It was shown in the above Figs. 4 and 5 that this latter process leads to hemispherical asymmetry. In this regard, it can be seen by comparison of top and bottom panels in Fig. 8 that the ion behaviors do significantly differ depending upon whether the particles originate from Northern or Southern Hemispheres. During the IMF change considered here (from $B_{\mathrm{X}}=0$ to $B_{\mathrm{X}}=20 \mathrm{nT}$ ), ions exhibit weaker nonadiabatic features in the Southern Hemisphere as compared to the northern one. Such an impulsive nonadiabatic transport at high latitudes likely forms a specific feature of the hermean environment because of both small field variation time scales (small $\tau_{B}$ ) and weak planetary magnetic field (large $\tau_{C}$ ).

Since the nonadiabatic response of the particles depends upon gyration period-to-field variation time scale ratio, one expects the dynamics of protons to significantly differ from that of $\mathrm{Na}^{+}$. This is illustrated in Fig. 10 that shows model $\mathrm{H}^{+}$trajectories during a similar IMF turning. Here again, it can be seen that, for the test protons reaching the equator 

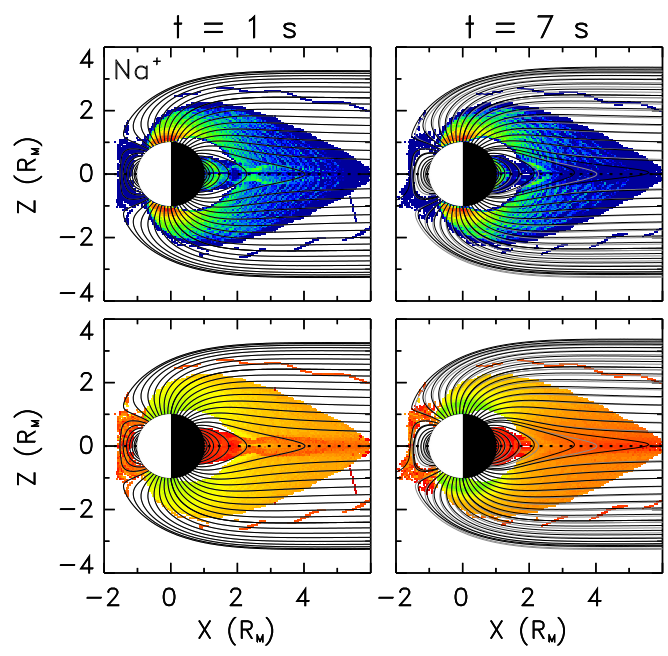

$t=7 \mathrm{~s}$

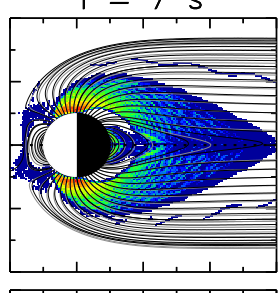

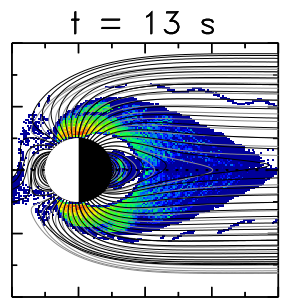

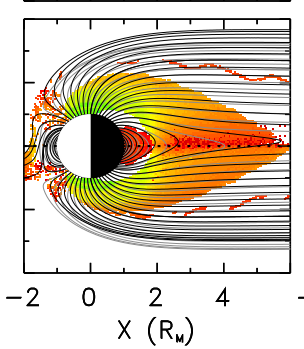

$t=19 \mathrm{~s}$

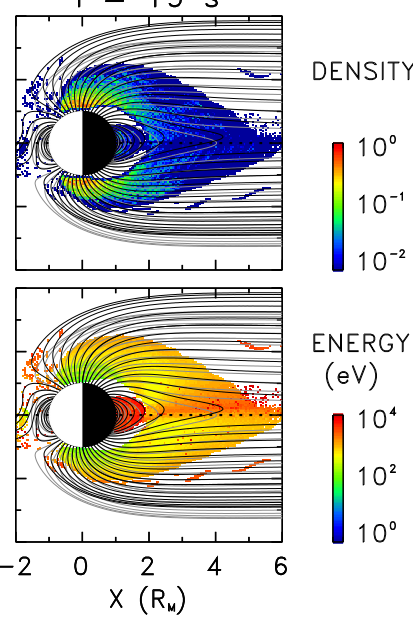

Fig. 11. (Top) Model normalized density and (bottom) average energy of $\mathrm{Na}^{+}$ions in the noon-midnight meridian plane during turning of the IMF (from $B_{X}=0$ to $B_{X}=20 \mathrm{nT}$ in $20 \mathrm{~s}$ ). The ions are launched from the high-latitude dayside sector (see text). The panels from left to right show snapshots at distinct times of the magnetic transition. Black and grey lines show the magnetic field lines at the corresponding time and in the initial configuration, respectively. Density and energy are coded according to the color scales at right.

before onset (trajectory coded in blue), similar results (e.g., similar trajectory apex) are obtained regardless of the hemisphere of origin. In contrast, for protons located away from equator at onset, the trajectories significantly differ depending upon the hemisphere of origin. Still, because of the much smaller gyroperiods of protons as compared to those of $\mathrm{Na}^{+}$, no significant $\mu$ change can be seen during IMF turning (right panels of Fig. 10), which contrasts with the results in Fig. 8.

To provide further insights into the response of ions to IMF turning, systematic trajectory computations were performed, considering test $\mathrm{Na}^{+}$launched from different altitudes (up to $100 \mathrm{~km}$ ) and latitudes (in the $50^{\circ}-90^{\circ}$ range) in both hemispheres of the dayside sector. The test $\mathrm{Na}^{+}$were initialized with $30^{\circ}$ pitch angle and an energy of $10 \mathrm{eV}$ comparable to that of exospheric neutrals (note that, due to the rapid centrifugal acceleration of the ions, little dependence was found upon these latter parameters). The test $\mathrm{Na}^{+}$were tracked according to a three-dimensional magnetospheric grid featuring bins of $0.05 R_{M}$ edge. Once the trajectory calculations in the initial steady state (IMF $B_{\mathrm{X}}=0$ ) were performed, each bin was assigned a test particle with specific weight that was traced throughout turning of the IMF (from $B_{X}=0$ to $B_{\mathrm{X}}=20 \mathrm{nT}$ in $20 \mathrm{~s}$ ). The density (normalized to the maximum value) and average energy in a given bin were subsequently calculated by summation over the various test ions occurring in that bin. The results of these computations are presented in Fig. 11 that shows the color-coded $\mathrm{Na}^{+}$density and energy at distinct times of the reconfiguration process.

It can be seen in the leftmost panels of Fig. 11 that the patterns in the initial state are similar to those of Fig. 6, with symmetrical flows above the polar cap and gradual cen- trifugal acceleration up to the keV range before entering the nightside plasma sheet. Most notably, moving from left to right in the bottom panels of Fig. 11, it is apparent that, in the course of the magnetic transition, the average energy of the $\mathrm{Na}^{+}$population in the magnetospheric lobes rapidly increases up to several keVs. As discussed above (see Fig. 8), the present energization occurs in a nonadiabatic manner as a result of resonance between the induced electric field and the ion gyromotion. Because this short-lived nonadiabatic energization depends upon gyroperiod versus field variation time scale, it preferentially affects heavy ions (e.g., $\mathrm{Na}^{+}$) and is negligible for protons.

In the different panels of Fig. 11, some ion flows with energies of several keVs can also be seen north and south of the main $\mathrm{Na}^{+}$population. Such flows result from peculiar ion drift paths as is illustrated in Fig. 12. It is apparent from this figure that, after transport into the nightside plasma sheet over the polar cap, the test $\mathrm{Na}^{+}$originating from the polar exosphere travels back into the dayside sector. It first mirrors at high latitude near dusk, then in the cusp region near noon at which point it is convected back over the pole into the lobe with an energy of several keVs. A similar circulation scheme was invoked in the study of Seki et al. (1998) (see, e.g., their Fig. 6) to explain the Geotail observations of energetic (several keVs) $\mathrm{O}^{+}$ions at large distances in the magnetospheric lobes. In addition, it is of interest to note that, at Mercury, the de-trapping zone of equatorial particles (leading to the so-called "Shabansky branching") extends over most of the dayside sector (shaded area in the bottom left panel of Fig. 12). That is, because of the existence of a field minimum at high latitude in the cusp region, the mirror force points away from equator near the frontside 

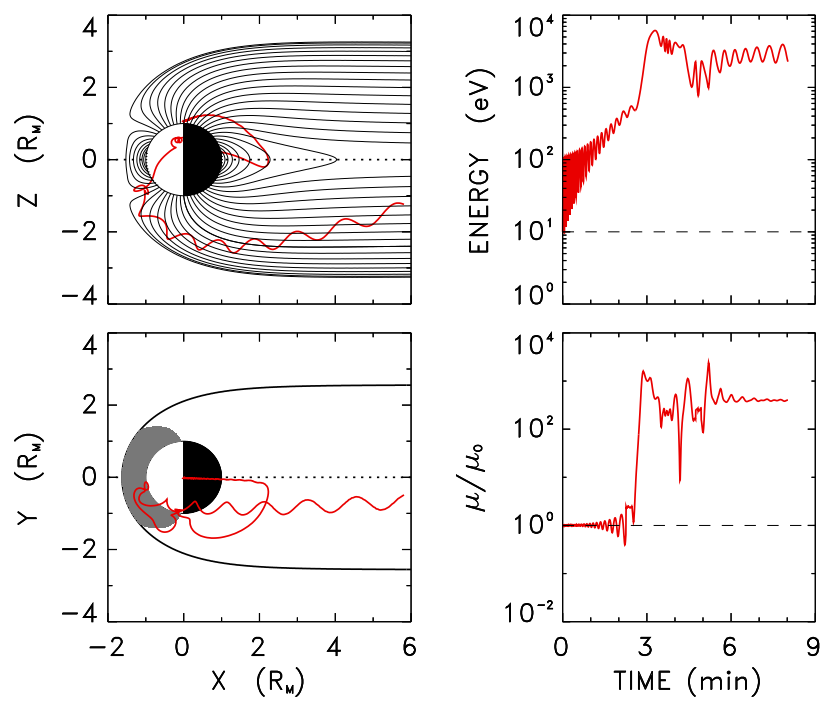

Fig. 12. Model $\mathrm{Na}^{+}$trajectory from polar exosphere to highlatitude magnetospheric lobe via nightside plasma sheet: (left) trajectory projections in the noon-midnight meridian plane and in the equatorial plane, (right) energy and magnetic moment (normalized to the initial value) as a function of time. In the bottom left panel, the thick line and shaded area show the magnetopause and the region of cuspward mirror force, respectively.

magnetopause, instead of equatorward elsewhere. Accordingly, (nearly) equatorially trapped particles are released toward high latitudes when they approach the frontside magnetopause, which provides a mechanism for populating the high-latitude region with energetic particles (Delcourt and Sauvaud, 1999). While at Earth, this de-trapping zone is limited to the very vicinity of the frontside magnetopause (see, e.g., Fig. 1 of Delcourt and Sauvaud, 1999), it extends over a wide domain at Mercury due to the small planetary field, hence hampering stable trapping at equator through most of the dayside magnetosphere.

\section{Conclusions}

The three-dimensional single-particle computations performed demonstrate that the IMF orientation has a prominent impact on the large-scale ion circulation at Mercury. A nonzero IMF $B_{\mathrm{X}}$ should lead to asymmetry between northern and southern magnetospheric lobes with a more pronounced $\boldsymbol{E} \times \boldsymbol{B}$ related centrifugal acceleration in one of them; hence, a more rapid filling of this very lobe and distinct accessible regions in the magnetotail depending upon the hemisphere of origin of the ions. The simulations also reveal that, in a similar manner to compression events (e.g., Delcourt et al., 2010), IMF turning at Mercury should lead to resonant heating of the ions under the effect of the induced electric field. That is, for ions that have gyration periods comparable to the field variation time scale, a possibly significant energization (up to the $\mathrm{keV}$ range and above) is obtained, characterized by prominent magnetic moment increase. Such a short-lived nonadiabatic heating of ions at high latitudes appears specific to the hermean environment that is characterized by a weak magnetic field as well as rapid field variations. During IMF turning, it should lead to hemisphere-dependent loading of the magnetospheric lobes with heavy energetic material, a feature that may be elucidated with the forthcoming MESSENGER data.

Acknowledgement. Topical Editor I. Daglis thanks P.-A. Lindqvist and another anonymous referee for their help in evaluating this paper.

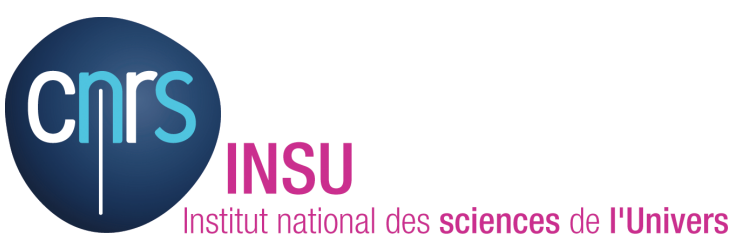

The publication of this article is financed by CNRS-INSU.

\section{References}

Anderson, B. J., Acuña, M. H., Korth, H., Purucker, M. E., Johnson, C. L., Slavin, J. A., Solomon, S. C., and McNutt Jr., R. L.: The structure of Mercury's magnetic field from MESSENGER's first flyby, Science, 321, 82-85, 2008.

Ashour-Abdalla, M., Bosqued, J.-M., El-Alaoui, M., Peroomian, V., and Walker, R.: Observations and simulations of a highly structured plasma sheet during northward IMF, J. Geophys. Res., 115, A10227, doi:10.1029/2009JA015135, 2010.

Büchner, J. and Zelenyi, L. M.: Regular and chaotic charged particle motion in magnetotaillike field reversals: 1 . Basic theory of trapped motion, J. Geophys. Res., 94, 94, 11821-11842, 1989.

Chen, J. and Palmadesso, P. J.: Chaos and nonlinear dynamics of single-particle orbits in a magnetotaillike magnetic field, J. Geophys. Res., 91, 1499-1508, 1986.

Cladis, J. B.: Parallel acceleration and transport of ions from polar ionosphere to plasma sheet, Geophys. Res. Lett., 13, 893-896, 1986.

Delcourt, D. C. and Sauvaud, J.-A.: Populating of the cusp and boundary layers by energetic (hundreds of $\mathrm{keV}$ ) equatorial particles, J. Geophys. Res., 104, 22, 635, doi:10.1029/1999JA900251, 1999.

Delcourt, D. C., Pedersen, A., and Sauvaud, J.-A.,: Dynamics of single-particle orbits during substorm expansion phase, J. Geophys. Res., 95, 20853-20865, 1990.

Delcourt, D. C., Sauvaud, J.-A., and Moore, T. E.: Polar wind ion dynamics in the magnetotail, J. Geophys. Res., 98, 9155-9169, 1993.

Delcourt, D. C., Moore, T. E., Orsini, S., Millilo, A., and Sauvaud, J.-A.: Centrifugal acceleration of ions near Mercury, Geophys. Res. Lett., 29, 32, doi:10.1029/2001GL013829, 2002.

Delcourt, D. C., Moore, T. E., and Fok, M.-C. H.: Ion dynamics during compression of Mercury's magnetosphere, Ann. Geophys. 28, 1467-1474, doi:10.5194/angeo-28-1467-2010, 2010. 
Heikkila, W. J. and Pellinen, R. J.: Localized induced electric field within the magnetotail, J. Geophys. Res., 82, 1610-1614, 1977.

Luhmann, J. G. and Friesen, L. M.: A simple model of the magnetosphere, J. Geophys. Res., 84, 4405-4408, 1979.

Moore, T. E., Fok, M.-C., Chandler, M. O., Chappell, C. R., Christon, S. P., Delcourt, D. C., Fedder, J., Huddleston, M., Liemohn, M., Peterson, W. K., and Slinker, S.: Plasma sheet and (nonstorm) ring current formation from solar and polar wind sources, J. Geophys. Res., 110, A010563, doi:10.1029/2004JA010563, 2005.

Ness, N. F.: The magnetosphere of Mercury, Solar system plasma physics, Vol. 2., Amsterdam, North-Holland Publishing Co., 1979.

Northrop, T. G.: The adiabatic motion of charged particles, Wiley Interscience, New York, 1963.

Peroomian, V. and El-Alaoui, M.: The storm-time access of solar wind ions to the nightside ring current and plasma sheet, J. Geophys. Res., 113, A06215, doi:10.1029/2007JA01287, 2008.

Russell, C. T. and Walker, R. J.: Flux transfer events at Mercury, J. Geophys. Res., 90, 11067-11074, 1985.

Seki, K., Hirahara, M., Terasawa, T., Mukai, T., Saito, Y., Machida, S., Yamamoto, T., and Kokubun, S.: Statistical properties and possible supply mechanisms of tailward cold $\mathrm{O}^{+}$ beams in the lobe/mantle regions, J. Geophys. Res., 103, 4477, doi:10.1029/97JA02137, 1998.
Slavin, J. A., Acuña, M. H., Anderson, B. J., Baker, D. N., Benna, M., Boardsen, S. A., Gloeckler, G., Gold, R. E., Ho, G. C., Korth, H., Krimigis, S. M., McNutt Jr., R. L., Raines, J. M., Sarantos, M., Schriver, D., Solomon, S. C., Trávníček, P., and Zurbuchen, T. H.: MESSENGER observations of extreme loading and unloading of Mercury's magnetic tail, Science, 329, 665668, 2010.

Speiser, T. W.: Particle trajectory in model current sheets, 1, Analytical solutions, J. Geophys. Res., 70, 4219-4226, 1965.

Volland, H.: A model of the magnetospheric convection electric field, J. Geophys. Res., 83, 2695-2699, 1978.

Zurbuchen, T. H., Raines, J. M., Gloeckler, G., Krimigis, S. M., Slavin, J. A., Koehn, P. L., Killen, R. M., Sprague, A. L., McNutt Jr., R. L., and Solomon, S. C.: MESSENGER observations of the composition of Mercury's ionized exosphere and plasma environment, Science, 21, 90-92, 2008. 